\title{
Treatment of Repeated Gastric Band Prolapse after Endoscopic Repair: A Case Report
}

\author{
SH Kang* and JS Yoo
}

Department of Surgery, Seoul Surgical Hospital, 109 Joongdaero, Songpa-gu, Seoul 05718, South Korea

Received: : August 23, 2016; Accepted: December 01, 2016; Published: December 05, 2016

*Corresponding author: SH Kang, Department of Surgery, Seoul Surgical Hospital, 109 Joongdaero, Songpa-gu, Seoul 05718, South Korea,

E-mail: animus70@snu.ac.kr

\begin{abstract}
Background: The Band prolapse complications of Laparoscopic Adjustable Gastric Banding (LAGB) can be treated endoscopically. We present two patients with repeated prolapses after endoscopic treatment who required gastric plication treatments.

Methods: From December 2007 to March 2016,1469 consecutive patients (227 male, 1242 female) underwent LAGB; 56 patients had band prolapses and 16 patients were treated by endoscopy. Among the 16 patients, two cases who showed repeated prolapses could be repaired with gastric plication treatments.

Results: The first case was of a 35-year-old woman underwent laparoscopic gastric plication due to repeated band prolapse. The second case was of a 43-year-old woman with a history of three episodes of band prolapse underwent gastric plication treatment. The clinical features are discussed.

Conclusions: Gastric plication treatment of recurred band prolapse is an effective and feasible procedure.

Keywords: Endoscopy; Band prolapse; Reduction; Gastric band; Gastric plication
\end{abstract}

\section{Introduction}

Laparoscopic Adjustable Gastric Banding (LAGB) is a simple, safe and effective procedure for treating morbid obesity. However, several complications after LAGB have been reported, such as band erosion, prolapse, gastric perforation, abscess, tube disconnection, port flip down and infection. These complications could be the main cause of failure after LAGB. For this reason, revisional weight-loss surgery after failed LAGB might be considered [1].

Band prolapse is a significant and common late complication after LAGB [2]. We have performed endoscopic treatment of band prolapse as we reported [3]. However, band prolapse occurred repeatedly in two cases. We present two patients with repeated prolapses after endoscopic treatment who required gastric plication treatments.

\section{Case Reports}

Case 1

A 35-year-old woman with BMI (body mass index) 40 underwent LAGB procedure to treat her obesity using the pars flaccida technique with no implication suture, and port placement under the anterior sheath of the rectus abdominis muscle (Figure1).

The patient presented a history of onset of band prolapse 22 months after the LAGB procedure (Figure 2). The symptoms

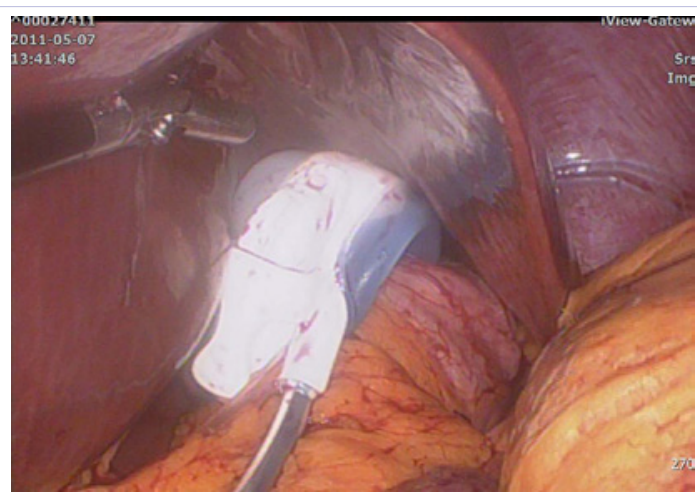

Figure 1: Pars flaccida technique without plication suture

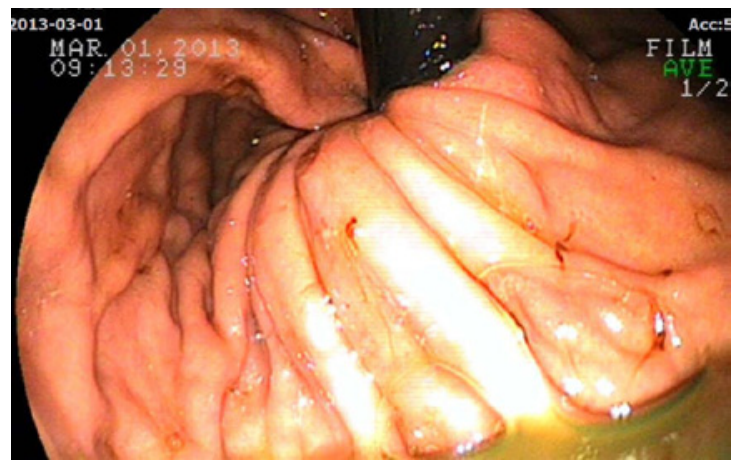

Figure 2: Endoscopic finding of gastric prolapse (First episode) 
of prolapse were sudden abdominal pain and repeated vomiting.

An endoscopic procedure was performed after deflation of the band under intravenous anesthesia. Band prolapse was diagnosed and treated by endoscopy. After a prolapsed stomach pouch was found, we inflated it with air. The prolapsed stomach pouch was gradually reduced as the stomach was inflated with air. The stomach was fully reduced and finally the band returned to its normal position. After reduction, the entire lumen of the stomach was examined to check its normality (Figure 3).

However, the patient had the second episode of band prolapse that required endoscopic treatment five months after the first one (Figure4). We treat edit with the same endoscopic technique as above (Figure 5).

The patient had recurrence that was treated by the endoscopic reduction technique 13 months after the second episode. The third endoscopic treatment of band prolapse was performed for an hour resulting in failure. She was sent to operating room and underwent laparoscopic reduction with two-row gastric plication(Figure16,17).The gastric greater curvature was plicated using 2/0 prolen from fundus at the level of diaphragm preserving the His angle to $10 \mathrm{cmproximal}$ to the pylorus. It took $70 \mathrm{~min}$ for the whole procedure with 3 trocars under general anesthesia. The patient was discharged in good condition 3 days after surgery. Fifteen months later, there was no evidence of recurrence (Figure 8).

\section{Case 2}

A 43-year-old woman with BMI 42 underwent LAGB procedure to treat her obesity using the pars flaccida technique with no implication suture, and port placement under the anterior sheath of the rectus abdominis muscle (Figure9).

The patient presented a history of onset of prolapse 10 months after the LAGB procedure (Figure 10). The symptoms of prolapse were sudden abdominal pain and repeated vomiting.

The endoscopic procedure was performed after deflation of the band under intravenous anesthesia. Band prolapse was diagnosed and treated by endoscopy. After a prolapsed stomach pouch was found, we inflated it with air. The prolapsed stomach was gradually reduced as the stomach was inflated with air. The

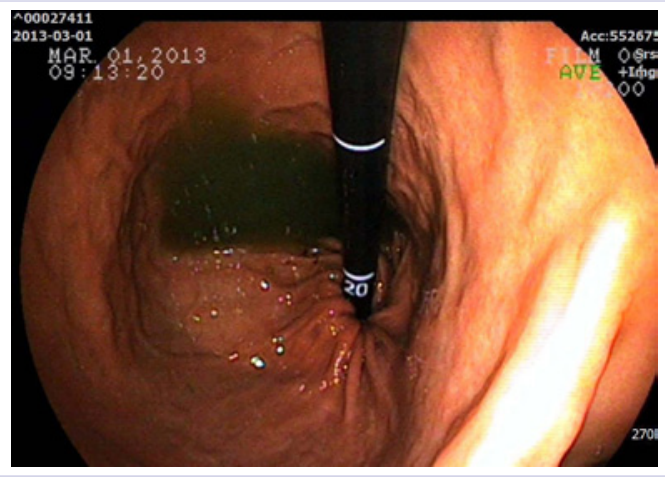

Figure 3: Endoscopic treatment of band prolapse

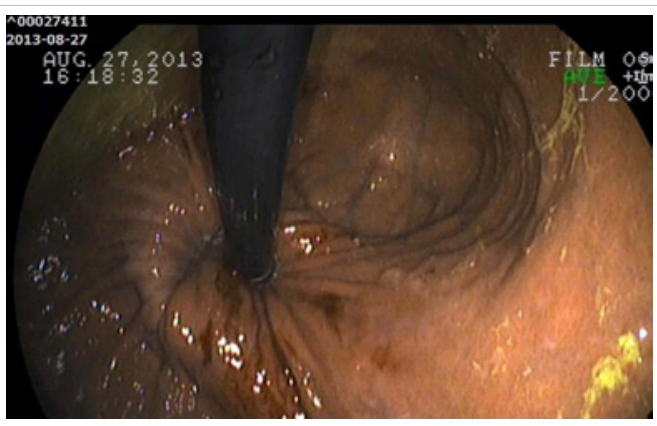

Figure 4: Endoscopic finding of second band prolapsed

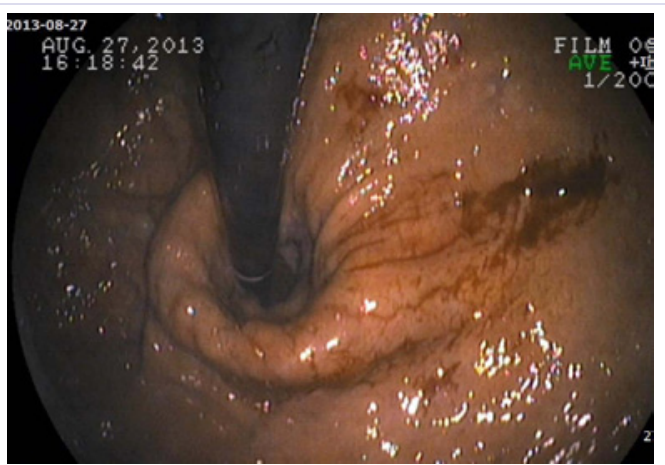

Figure 5: Endoscopic treatment of recurred band prolapse

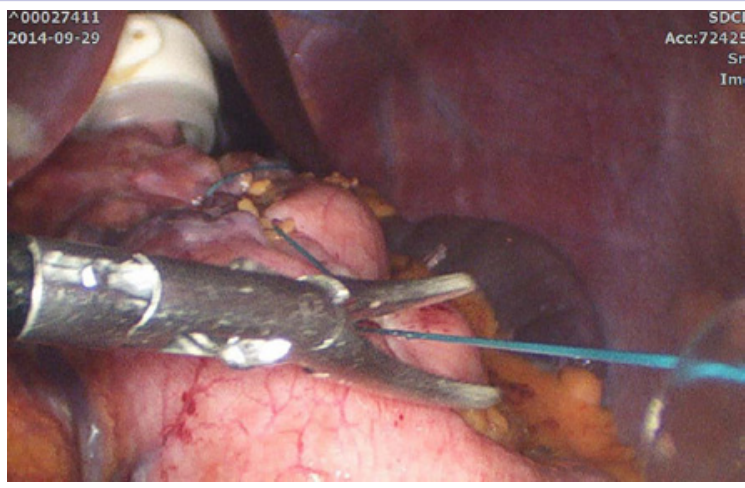

Figure 6: Operative reduction of band prolapse

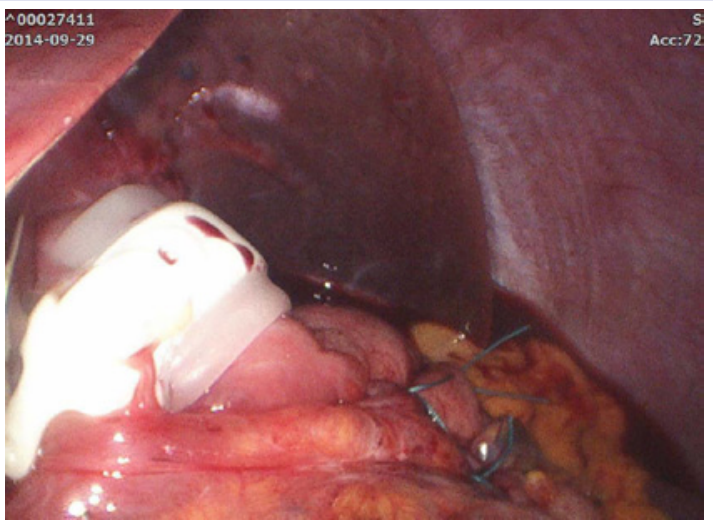

Figure 7: Gastric plication along the greater curvature of stomach 
stomach was fully reduced and finally the band returned to its normal position. After reduction, the entire lumen of the stomach was examined to check for normality (Figure 11).

However, this patient had the second and third episode of band prolapse which required endoscopic treatment 4 months after the first and second reduction respectively (Figure12,13). We treated it with the same endoscopic technique as above (Figure 14, 15).

The patient had the fourth band prolapse one month after the third episode. The endoscopic treatment of band prolapse was performed for an hour resulting in failure. She was sent to operating room and underwent laparoscopic reduction with one-row gastric plication (Figure16,17).The gastric greater curvature was plicated using $2 / 0$ prolen from fundus at the level of diaphragm preserving the His angle to $10 \mathrm{cmproximal}$ to the pylorus. It took $75 \mathrm{~min}$ for this procedure with 3 trocars under general anesthesia. The patient was discharged in good condition 3 days after surgery. Five months later, there was no evidence of recurrence (Figure 18).

\section{Discussion}

Since its first introduction in 1993, LAGB has been accepted as a reliable surgical option for treating obesity thanks to its safety, minimal invasiveness and effectiveness. However, bariatric surgeons have largely abandoned LAGB since 2008

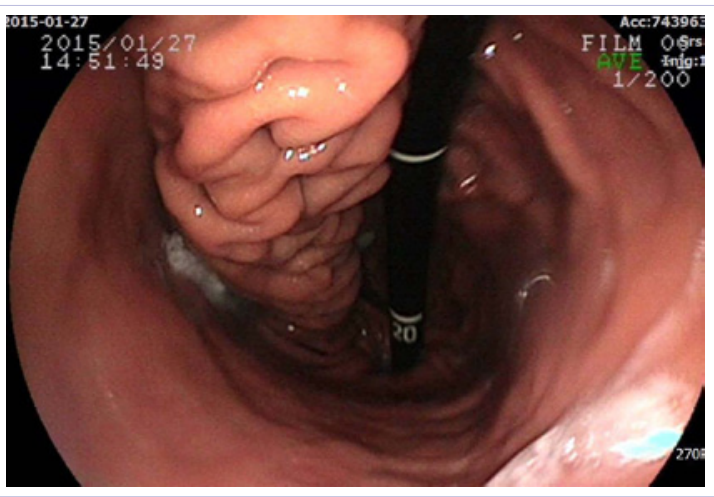

Figure 8: Endoscopic finding of gastric plication

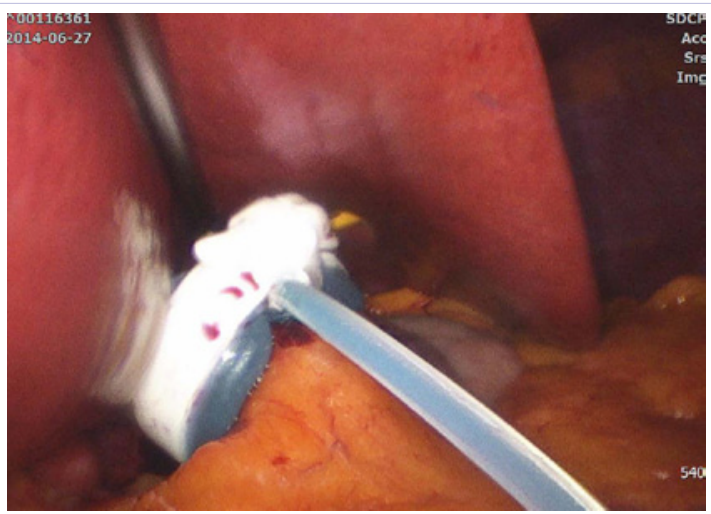

Figure 9: Pars flaccida technique without plication suture

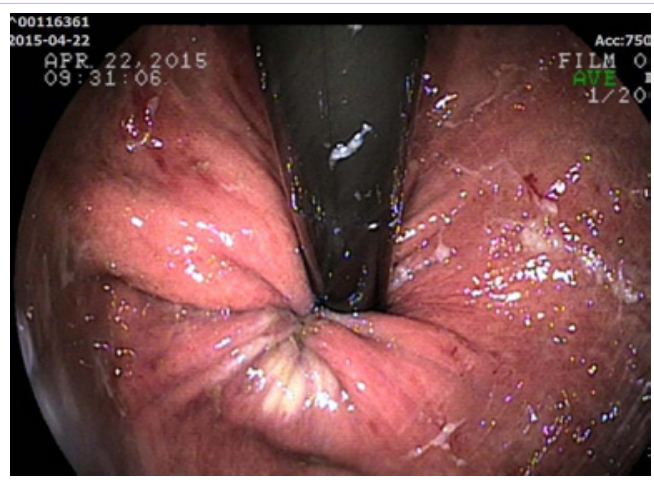

Figure 10: Endoscopic finding of gastric band prolapsed

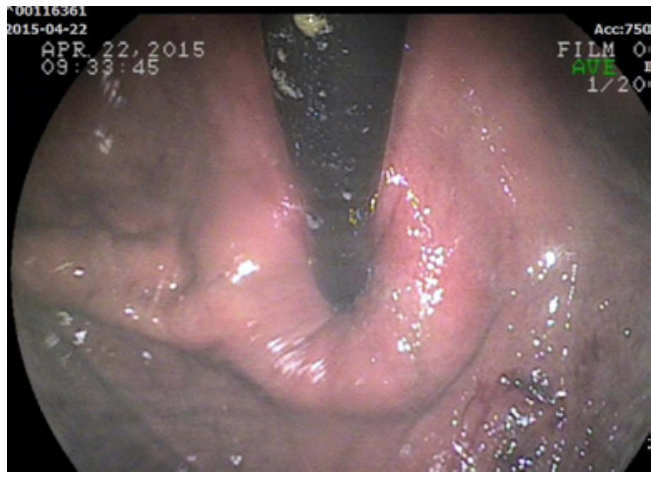

Figure 11: Endoscopic repair of the band prolapsed

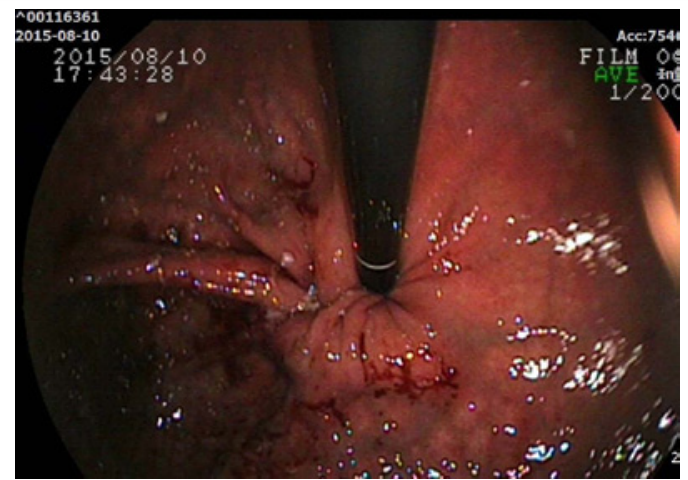

Figure 12: Second band prolapsed

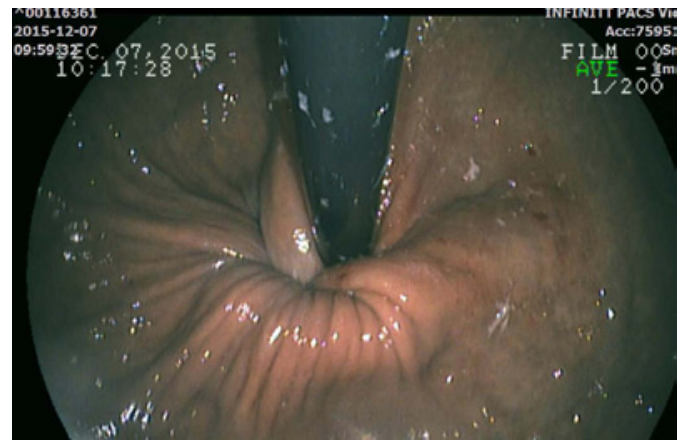

Figure 13: Third band prolapsed

Citation: Kang SH, Yoo JS (2016) Treatment of Repeated Gastric Band Prolapse after Endoscopic Repair: A Case Report. Obes Control

Page 3 of 5

Ther 3(1): 1-5. DOI: http://dx.doi.org/10.15226/2374-8354/2/2/00125 


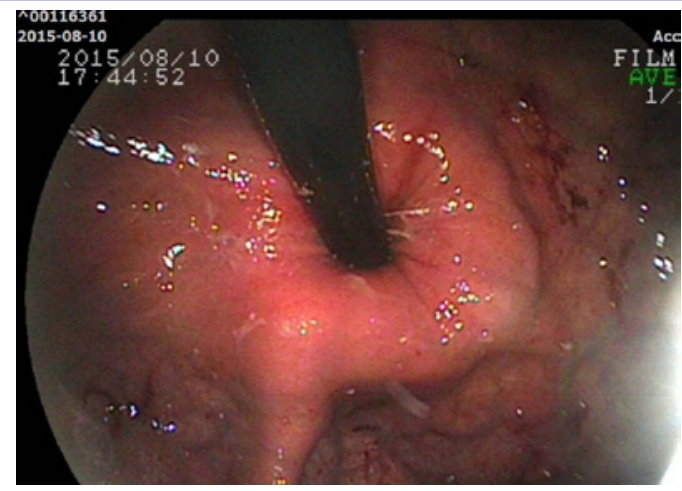

Figure 14: Second endoscopic reduction of band prolapse.

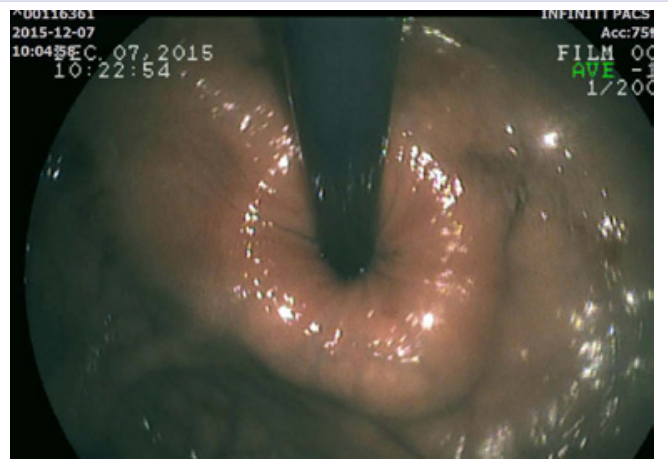

Figure 15: Third endoscopic reduction of band prolapse

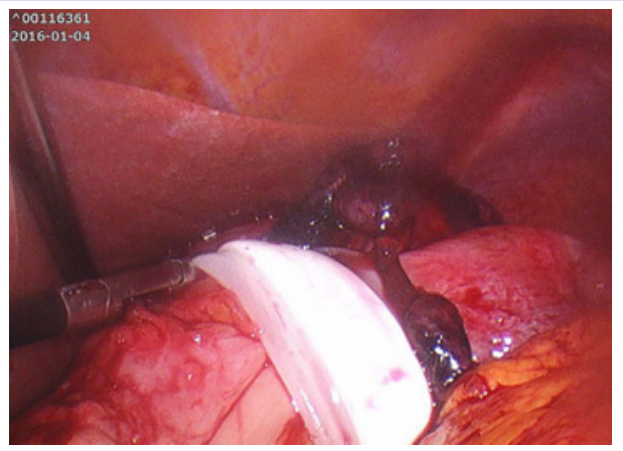

Figure 16: Operative reduction of band prolapse

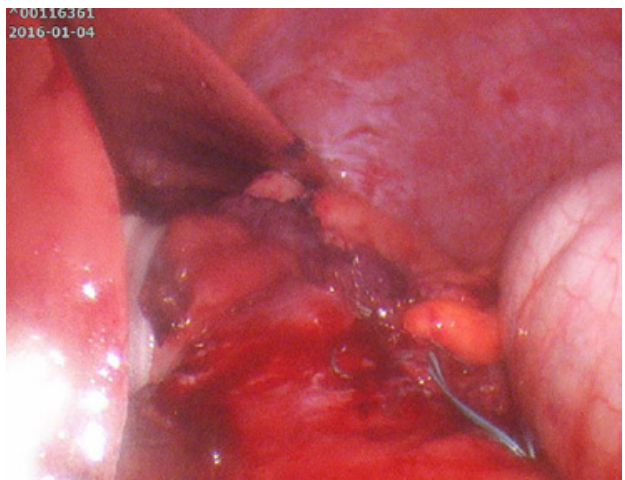

Figure 17: Gastric plication along the greater curvature of stomach

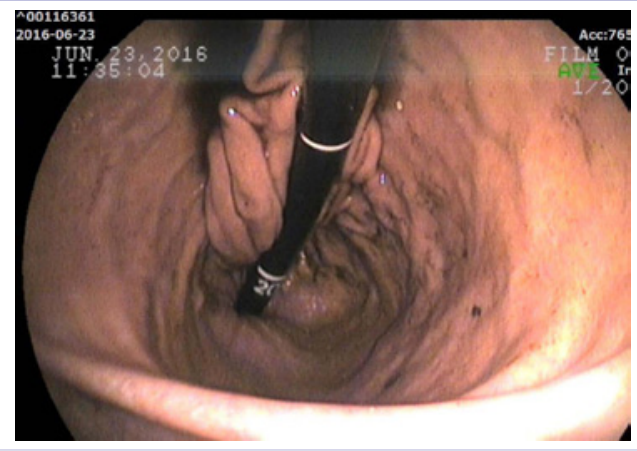

Figure 18: Endoscopic finding of the gastric plication

and the number of procedures has declined because of adverse complications such as band erosion or prolapse [4]. Surgical techniques have been suggested to lower the incidence of band prolapse, such as reduction in the size of the gastric pouch, the appropriate placement of gastro gastric sutures and correct positioning of the posterior aspect of the band. The main advantage of gastro gastric suturing in LAGB is to prevent band prolapse [5,6].

Band prolapse is a significant and common late complication after LAGB. The incidence of band prolapse within the first 5 years is still as much as $5 \%$, requiring operative procedures. It can occur anteriorly or posteriorly. The common symptoms and signs of band prolapse include food intolerance, vomiting, abdominal pain, reflux esophagitis, pouch dilatation, dehydration and muscle weakness [7].

This can be diagnosed by X-ray with a barium contrast medium or by endoscopy $[8,9]$. A delay in diagnosis can cause hypokalemia with or without loss of consciousness, gastric perforation or pouch necrosis leading to death [10]. As band prolapses do not respond to conservative measures, many surgeons advocate surgical options including repositioning of the band, replacement with a new one or even removal of the band [11].

We evaluated the stomach by endoscopy before surgery and every 6 months after the LAGB procedure, and when the patients had symptoms of prolapse such as sudden abdominal pain and repeated vomiting.

An endoscopic procedure was performed after deflation of the band when the patient presented these symptoms of prolapse. When diagnosed for band prolapse, the patient was sent to the endoscopy room under intravenous anesthesia. If we found a prolapsed stomach pouch, we inserted an endoscope and then inflated it with air. The prolapsed stomach pouch was gradually reduced as the stomach was inflated with air. The stomach was fully reduced and finally the band returned to its normal position. After reduction, we examined the entire lumen of the stomach to check on its normality.

Not all anterior band prolapses have reduced by band deflation and endoscopic approach. When we found any sign of infection or perforation inside the stomach, we fixed it 
operatively. Otherwise, almost all the band prolapses after such suture less LAGB without delay in diagnosis can be fixed easily with an endoscopic procedure. Those patients with repeated band prolapses could be treated by one-row or two-row plication technique. The gastric greater curvature was plicated using $2 / 0$ prolen from fundus at the level of diaphragm preserving the His angle to $10 \mathrm{cmproximal}$ to the pylorus. The aim of the plication was restriction of the prolapsed portion of the stomach via folds from its own wall [12]. However, we need long term follow-up to evaluate the efficacy of the gastric plication and endoscopic treatment of the band prolapse.

\section{Conclusion}

Even though endoscopic treatment of the band prolapse is safe and feasible, two patients with repeated band prolapse were treated with laparoscopic gastric plication technique. We need long-term follow up to evaluate the efficacy of the gastric plication and endoscopic treatment of the band prolapse.

\section{Conflict of Interest}

ll authors Sehoon Kang and Jisook Yoo have no conflicts of interest or financial ties to disclosure.

\section{References}

1. Tran TT, Pauli E, Lyn-Sue JR, Haluck R, Rogers AM. Revisional weight loss surgery after failed laparoscopic gastric banding: an institutional experience. Surg Endosc. 2013;27(11):4087-93. doi: 10.1007/ s00464-013-3065-6.

2. Brown WA, Burton PR, Anderson M, Korin A, Dixon JB, Hebbard G, O'Brien PE. Symmetrical pouch dilatation after laparoscopic adjustable gastric banding: incidence and management. Obes Surg. 2008;18(9):1104-8. doi: 10.1007/s11695-008-9485-z.
3. SH Kang, KC Kim, KH Kim. Endoscopic treatment of gastric band prolapse. Obes Surg. 2014;24(6):954-7. doi: 10.1007/s11695-0141253-7.

4. Buchwald H, Oien DM. Metabolic/bariatric surgery worldwide 2011. Obes Surg. 2013;23(4):427-36. doi: 10.1007/s11695-012-0864-0.

5. Favretti F, Cadière GB, Segato G, Himpens J, De Luca M, Busetto L, et al. Laparoscopic banding: selection and technique in 830 patients. Obes Surg. 2002;12:385-90.

6. Fielding GA, Allen JW. A step-by-step guide to placement of the LAP-BAND adjustable gastric banding system. Am J Surg. 2002;184(6B):26S-30S.

7. Favretti F, Cadiere GB, Segato G, Himpens J, Busetto L, De Marchi F, et al. Laparoscopic adjustable silicone gastric banding (LAP-BAND): how to avoid complications. Obes Surg. 1997;7:352-58.

8. Mortelé KJ, Pattijn P, Mollet P, Berrevoet F, Hesse U, Ceelen W, et al. The Swedish laparoscopic adjustable gastric banding for morbid obesity: radiologic findings in 218 patients. AJR Am J Roentgenol. 2001;177(1):77-84

9. Toppino M, Cesarani F, Comba A, Denegri F, Mistrangelo M, Gandini G, et al. The role of early radiological studies after gastric bariatric surgery. Obes Surg. 2001;11(4):447-54.

10. Chevallier JM, Zinzindohoué F, Elian N, Cherrak A, Blanche JP, Berta $\mathrm{JL}$, et al. Adjustable gastric banding in a public university hospital: prospective analysis of 400 patients. Obes Surg. 2002;12(1):93-9.

11. Hadar S, Franco F. Avoiding postoperative complications with the LAP-BAND system. Am J Surg. 2002;184(Suppl):31S -37S.

12. Talebpour M, Motamedi SM, Talebpour A, Vahidi H. Twelve year experience of laparoscopic gastric plication in morbid obesity: development of the technique and patient outcomes. Ann Surg Innov Res. 2012;6(1):7. doi: 10.1186/1750-1164-6-7. 